\title{
Key cognitive preconditions for the evolution of language
}

\author{
Merlin Donald $^{1}$
}

Published online: 1 July 2016

(C) Psychonomic Society, Inc. 2016

\begin{abstract}
Languages are socially constructed systems of expression, generated interactively in social networks, which can be assimilated by the individual brain as it develops. Languages co-evolved with culture, reflecting the changing complexity of human culture as it acquired the properties of a distributed cognitive system. Two key preconditions set the stage for the evolution of such cultures: a very general ability to rehearse and refine skills (evident early in hominin evolution in toolmaking), and the emergence of material culture as an external (to the brain) memory record that could retain and accumulate knowledge across generations. The ability to practice and rehearse skill provided immediate survival-related benefits in that it expanded the physical powers of early hominins, but the same adaptation also provided the imaginative substrate for a system of "mimetic" expression, such as found in ritual and pantomime, and in proto-words, which performed an expressive function somewhat like the home signs of deaf non-signers. The hominid brain continued to adapt to the increasing importance and complexity of culture as human interactions with material culture became more complex; above all, this entailed a gradual expansion in the integrative systems of the brain, especially those involved in the metacognitive supervision of self-performances. This supported a style of embodied mimetic imagination that improved the coordination of shared activities such as fire tending, but also in rituals and reciprocal mimetic games. The time-depth of this mimetic adaptation, and its role in both the construction and acquisition of languages, explains the importance of mimetic expression in the media, religion, and politics. Spoken
\end{abstract}

Merlin Donald

donaldm@queensu.ca

1 Professor Emeritus, Psychology Department, Queen's University, Kingston, ON K7L 3N6, Canada language evolved out of voco-mimesis, and emerged long after the more basic abilities needed to refine skill and share intentions, probably coinciding with the common ancestor of sapient humans. Self-monitoring and self-supervised practice were necessary preconditions for lexical invention, and as these abilities evolved further, communicative skills extended to more abstract and complex aspects of the communication environments - that is, the "cognitive ecologies"-being generated by human groups. The hominin brain adapted continuously to the need to assimilate language and its many cognitive byproducts by expanding many of its higher integrative systems, a process that seems to have accelerated and peaked in the past half million years.

Keywords Cognitive neuroscience $\cdot$ High order cognition

There is no reason to think that language evolved directly, that is, as language. Rather, it is the long-term interactive product of a very early [ca. 3.5 million years ago (Mya)] and unique shift in the direction of human cognitive evolution, marked by two important developments. The first was the emergence of a general, supra-modal capacity to rehearse and refine skills; and the second was the emergence of material culture as a major force shaping the direction of human cognitive evolution.

Evidence for the former, refined skills, appears in the archaeological record of australopithecines. They were using stone tools to butcher game long before the genus Homo came into existence (McPherron et al., 2010). These early artifacts first took the form of Oldowan, and then Acheulian stone tools, both of whose manufacture is out of the reach of modern chimpanzees and gorillas (Toth, Schick, \& Semaw, 2003).

These artifacts provide good evidence that there was a major change in primate motor learning capacity early in the 
Pleistocene epoch that enabled the deliberate modification of procedural memories. This important advance had implications that went far beyond the manufacture of stone tools. It established a key precondition for the future evolution of more sophisticated expressive systems, including languages, which require a far more powerful, but qualitatively similar, capacity to refine and rehearse skill sets. It also started, however modestly at first, a hominin process of externalizing memory, that is, using the material cultural products of skill to build a record of past achievements and knowledge that could exist external to the brain, and be carried forward for future generations.

This latter development was a key factor in driving a process whereby brain and culture could co-evolve, with hominin brain design increasingly falling under the evolutionary sway of its vital relationship with an evolving range of material artifacts. Viewed retrospectively, these two changes, refined skills, and a cumulative memory record external to the brain that was a product of those skills, might be regarded as a necessary pre-adaptation that enabled the later evolution of language. They opened up the possibility of building a material memory bank that could evolve into a much more complex ecology for cognitive development. However, there is no reason to think that they were specific to language, even in truncated form; on the contrary, these early changes reflected a very basic evolutionary agenda, tied primarily to the immediate survival value of extending the physical capacities of hominins with improved material artifacts.

The presence of material culture also transformed early hominin social groups into simple distributed cognitive systems, in which skills and artifacts were distributed across groups of brains, and embedded in networks of practice that combined organic components (brains) with inorganic ones (a modifiable ecology of artifacts) in small social groups. This injected a new possibility into the adaptive equation: a brain embedded in a co-evolutionary relationship with a distributed system would be subjected to a different set of selection pressures, not only because group learning capacity would become paramount under such circumstances, but because the evolving cognitive system could also "offload" crucial epigenetic steps to the evolving distributed network. The direction of evolution could thus shift gradually away from evolving fully equipped, largely self-contained individuals, toward interactive and social capacities that would ensure the successful operation of the social network as a distributed cognitive system.

In my own work, I have developed a gradualistic coevolutionary scenario for language based upon these kinds of early changes to the primate cognitive repertoire (Donald, 1991, 1993a, 1999, 2001, 2013).

1. Languages emerge from the interactions of several brains in social networks, and there is no evidence for the idea that, on its own, a single isolated human brain could generate, or even conceive of, language. Brains get their languages from elsewhere. Languages are negotiated, like treaties, in a communicative environment, by brains that are completely non-symbolic (as in "neural net" computation) in their internal operations. Following that logic, the intricate complexities of language, including even such exquisite phenomena as syntax, reflect the complexity of the challenges presented by the interactive environment that generated it. As in the case of visual experience, the complexity of language is imposed from outside.

2. If languages are products of cognitive interactions in groups, this fact alone would demand a culture-first theory of language genesis. The evolutionary question then becomes: how would sophisticated and cognitively demanding interactive environments (complex cultures) have evolved in the first place? In such a scenario, our focus should shift to describing what adaptations to the hominid brain would be essential for consolidating the complex forms of culture that would trigger the spontaneous combustion of such exotic things as languages.

3. Another, and closely related, point follows: languages are not the only evolutionarily novel aspects of the human mind that demand a special evolutionary explanation. Archaeological evidence suggests strongly that human ancestors were skilled long before they were articulate. Of course, the truth of this assertion depends upon our definition of language, but there is no evidence that full language capacity - defined as the ability to tell an original story based on personal experience (Nelson, 1998)_existed prior to the emergence of sapient humans, whereas rehearsed and refined skills-sufficiently complex that they required at least some pedagogical guidance for their transmission (Morgan et al., 2015)_existed millions of years earlier. Refined skill has its own very clear adaptive rationale, but it may also have set the stage, serving as a precondition for the kind of cultural evolution that could eventually produce language.

4. Of course, the possibility must be entertained that language came first, and was the precondition for complex culture. However, that hypothesis has a fatal flaw: languages are complex skill-hierarchies in themselves, and must be learned. Therefore the cognitive apparatus for refining skill must have existed in some form before languages could emerge from group interactions. There is a causal chain argument at the core of this argument: refined skill, involving the systematic practice and rehearsal of an action toward improving its outcome, is a requirement for lexical invention. Humans have a remarkable capacity for acquiring, rehearsing, and refining hundreds of skills, and arranging them in complex automatized hierarchies; language is just one example of 
such a hierarchy. I have argued that a general capacity for refining skill was fundamental because a capacity for refining skill is a logical precondition for the evolution of languages: any morpho-phonology, and any lexicon, whether oral, manual, or multimodal, consists of a complex set of conventionalized and automatized skills (Donald, 1998b). Thus the evolution of a capacity for refining complex skill-hierarchies is a prior consideration in any theory of language evolution. A parsimonious theory should try to account for both features, that is, for both refined skill and language, in that order.

5. The convergence of refined skill and a much more complex material culture in the Lower Paleolithic, and possibly earlier, is evidence that hominin cognitive evolution had already turned in a uniquely collective direction. The effective use of refined skills in groups entails both some degree of pedagogy and collaboration; recent work by Morgan et al. (2015) confirms this to be the case, even in anatomically modern humans attempting to improve their Oldowan toolmaking skills. Survival was increasingly linked to the individual's ability to interact effectively with some form of a networked public memory system, however primitive, exemplified perhaps by the networks of practice that still characterize cultural transmission systems for preserving skill in human populations (Hutchins, 1995). Memory for a variety of special skills usually involves some division of labor, as well as a collaborative strategy for passing those skills on to every new generation. Thus the memories that sustained a skilled society of hominins were held collectively, aided by the existence of material reminders of the ways of life of past generations.

6. This arrangement provided a framework for subsequent adaptations to a variety of new and different ecologies. This lifestyle was necessarily supported by conventionalized networks of practice, which by their very nature, acquire significance, forming a foundation for a "mimetic" system of expression that includes conventionalized gestures, group displays and rituals, and proto-words. This kind of culture shares much in common with cultures observed in other mammals (see, for example, Whiten, 2011), but human mimesis has the added element of less fixed stereotypy, much wider and innovative variety, and greater flexibility across expressive modalities, as well as that important link to potentially cumulative material cultural change.

7. Mimetic performances are somewhat similar in their semiotic function to the home signs of deaf people who lack sign language (see Goldin-Meadow \& Brentari, 2015; also Donald, 2001, chapter 6). This "mimetic" form of culture marked the first truly public representations of hominin culture, characterized by an expressive mode best labelled as "embodied action-metaphor." This mode of expression has been retained in modern human society, not only in the transmission of skill, but also in such common phenomena as public displays of grief, power, and celebration; and in the implicit expressive codes found in athletics, dance and song, as well as some aspects of theatrical performance (Donald, 1998a, 2001, 2013).

8. The move to mimetic culture was an essential first step in the direction of language. It created a communicative culture that was capable of accumulating knowledge (mostly about making and using tools in its earliest stages, but also about gesture and ritual) in a distributed cognitive-cultural memory system. Since group behavior "records" and preserves the rules of even the simplest conventions, individual brains were relieved of the need to be the sole source for remembering the operations needed to reproduce any specific suite of hominin survival skills.

9. This development also changed the direction of brain evolution in an important way: complex skills require a high degree of metacognitive self-supervision and social awareness. Archaeological studies of fire use in human ancestors provides even more compelling evidence that Homo erectus was moving in that direction over $1 \mathrm{Mya}$ (Berna et al., 2012; Wrangham, 2009). There is (as yet) no evidence that this level of self- supervision existed in the australopithecines, whose toolmaking skills were more limited, and nonhuman primates do not rehearse skills at all. This provides an important comparative guidepost to what is unique in the human capacity for refining skill. Nonhuman primates, including presumably our Miocene common ancestor, seem to lack the kind of detailed and flexible self-representational maps needed to guide the sophisticated rehearsal and refinement of action. Even after having being taught how to break stones to create a cutting tool, bonobos do not try to improve their technique. Similarly, although gibbons may throw stones as projectiles in regional fights over territory, they never think to practice or refine their technique. The necessary metacognitive capacities are underdeveloped in these species, but were evidently highly developed in the human line (Donald, 2013), and especially evident after the emergence of Homo erectus. Given their ability to fashion the simpler forms of finished stone tools, australopithecines probably fell somewhere between the Miocene primates and later hominins.

10. Much more systematic and extensive rehearsal is necessary to master the techniques involved in manufacturing Acheulian tools, especially those made from the hardest, sharpest substances available, flint and obsidian. In rehearsing such finely tuned patterns of action, actors must first recall their previous attempts with some precision, 
and then reproduce those accurately, with modifications, while evaluating the effectiveness of each attempt to reach an idealized result.

11. That sequence - the review-rehearsal loop-depends upon a rudimentary form of voluntary memory retrieval. The existence of more complex stone tools such as the Acheulian hand axe is solid evidence that a change had already occurred in the brain of Homo erectus, enabling not only improved metacognitive self-supervision, but also more accurate recall of very specific procedural memories, along with detailed evaluation of performance outcomes. This development seems to have reached a critical point approximately 1.6 Mya, with the appearance of the first Acheulian toolkits made from flint. Their effective use was responsible for a hominin "master toolkit" that could be used to make and refine other kinds of tools from a variety of softer materials (Donald, 1993b, 1998b).

12. The gradual expansion of the hominin toolkit, along with an increasing number of cultural achievements, including the mastery of fire and the cooking of food, provides evidence of an evolving capacity for the ability to rehearse and refine more complex skills by circa 500,000 years ago (Kya). The skills involved in fashioning a tool such as a handaxe or a hafted spear entail mastering a hierarchy of several interlinked and automatized sub-routines in a wider context (see Whiten, Toth \& Schick, 2009). Autocuing, or the ability to deliberately search one's own memory, must become increasingly accurate and fast as skills become more complex. Because of its exquisite context-dependency, the capacity for autocuing procedural memories provided a platform for evolving a path to mediate recall of other kinds of memories, including those that are central to the modern human mind, such as semantic and episodic memory (Donald, 2012).

13. The unique vocal skills of hominins are an additional evolutionary puzzle that waits for a detailed solution. I suggested, based on a review of the neuropsychological evidence available at that time (1991), that the "review-rehearsal loop" underlying refined skills was a supra-modal adaptation from the start, engaging the entire pre-existing Miocene primate voluntary motor repertoire, including whatever limited voluntary vocal capacities primates already had. Under those conditions, selection pressures favoring an expanded vocal range would have developed wherever and whenever an increased vocal range proved adaptive, opening the door to gradual "voco-mimetic" evolution in parallel with the review-rehearsal loop. This might have started quite early in the hominin scenario, as early as 3.5 Mya, but was almost certainly underway by the time Acheulian tool culture first emerged around 1.6 Mya. Paleogenetic research might eventually resolve this issue.

14. Mimesis, including voco-mimesis, may have set the stage for the emergence of more complex communication, but other variables also needed to be in place for the emergence of speech, including high speed vocomimetic recall. A vocal language of any kind demands a flexible and highly efficient system for high-speed online autocuing of selected lexical items stored in an expanded procedural memory system (see Donald, 2012).

15. Moreover, as will undoubtedly be spelled out in detail by other contributors to this volume, the evolution of joint intentionality, leading to the elaborate and detailed mindsharing cultures of the modern era, and increasingly intricate intersubjective representational games, was an additional precondition for the final evolutionary push in the direction of full-fledged language (Tomasello, 2008). The strictest developmental criterion for full language capacity is the ability to construct an original narrative based on personal experience (Nelson, 1998). This criterion has obviously been met by anatomically modern humans; it is not yet clear whether it was met by other closely related hominins.

16. There will be no quick fix or easy road to a more detailed theory of language evolution. A full theory will require good evidence on exactly what changed, and when, in the hominid brain during the lead-up to the point where human ancestors were able to spontaneously generate languages. An adequate level of theoretical detail cannot be achieved without further advances in paleo-genetics, and a considerably more advanced model of the detailed functional architecture of the cerebral cortex, than anything currently available.

17. Nevertheless, we have come a long way towards a general explanation in principle. Rather than evolving in a direct manner, or as a specialized modular system added to a primate brain, language seems to have emerged as a cultural phenomenon, after a graduated series of modifications to a set of pre-existing, and basically primate, cognitive capacities that created the unique preconditions needed for the genesis of a collective, distributed and shared system of thought and memory, which eventually led to spoken languages and other, literacydependent symbolic systems in the complex multilayered mix found in modern humans.

18. Language is, in this sense, not a feature of the brain per $s e$. It is a cognitive epiphenomenon, a socially constructed (Searle, 1969) cultural over-write imposed on a brain which is essentially primate in its design. Language thus has its origin in a distributed cognitive system, while it is performed by a local cognitive system, that is, the brain of an individual. It is the child of an interactive cultural imagination, that is, of groups of brains in collision. Its 
original interactive logic is primarily "mimetic" in the Aristotelian sense, that is, it follows an analogue, embodied logic, based on a greatly expanded capacity for procedural learning and mimetic expression. Purely symbolic expressive systems such as analytic and mathematical thought eventually emerged from imposed sets of culturally generated conventions and algorithms, but they were deliberate, highly interactive, and thus entailed a heavy load on conscious capacity in their invention (see Donald, 2001). But these innovations were developed only very recently, when viewed in an evolutionary context; and only emerged after long experience with artificial symbolic devices such as mathematical notations. The primary evolutionary process driving language evolution was a broadly cognitive one, not a specifically linguistic one. It involved the simultaneous juggling of many different brain parameters (including plasticity itself) which co-evolved with a host of interactive cultural parameters, under the profound influence of the rich material culture that has defined the survival strategy of genus Homo.

\section{References}

Berna, F., Goldberg, P., Horwitz, L.K., Brink, J., Holt, S., Bamford, M., \& Chazan M. (2012). Microstratigraphic evidence of in situ fire in the Acheulean strata of Wonderwerk Cave, Northern Cape province, South Africa. Proceedings of the National Academy of Sciences of the United States of America, 109, E1215-E1220. doi: 10.1073 /pnas.1117620109

Donald, M. (1991). Origins of the modern mind: three stages in the evolution of culture and cognition. Cambridge, MA: Harvard University Press.

Donald, M. (1993a). Précis of Origins of the Modern Mind with multiple reviews and author's response. Behavioral and Brain Sciences, 16, 737-791.

Donald, M. (1993b). Hominid enculturation and cognitive evolution. Archaeological Review from Cambridge, 12, 5-24.

Donald, M. (1998a). Material culture and cognition: concluding thoughts. In C. Renfrew \& C. Scarre (Eds.), Cognition and culture: the archaeology of symbolic storage (pp. 181-187). Monographs of the McDonald Institute for Archaeological Research: University of Cambridge, U.K.
Donald, M. (1998b). Mimesis and the executive suite: missing links in language evolution, pp. 44-67. In J. R. Hurford, M. StuddertKennedy, \& C. Knight (Eds.), Approaches to the evolution of language: social and cognitive bases. Cambridge, MA: Cambridge University Press.

Donald, M. (1999). Preconditions for the evolution of protolanguages, pp. 120-213. In M. C. Corballis \& I. Lea (Eds.), The Descent of Mind. Oxford: Oxford University Press.

Donald, M. (2001). A mind so rare: the evolution of human consciousness. New York: Norton.

Donald, M. (2012). Evolutionary origins of autobiographical memory, pp. 269-289. In D. Berntsen \& D. C. Rubin (Eds.), Understanding autobiographical memory: themes and approaches. New York: Cambridge University Press.

Donald, M. (2013). Mimesis theory re-examined, twenty years after the fact, pp. 169-192. In G. Hatfield \& H. Pittman (Eds.), The evolution of mind, brain and culture. Philadelphia: University of Pennsylvania Press.

Goldin-Meadow, S., \& Brentari, D. (2015). Gesture, sign and language: the coming of age of sign language and gesture studies. Behavioral and Brain Sciences. doi:10.1017/S0140525X15001247

Hutchins, E. (1995). Cognition in the Wild. Boston, MA: MIT Press.

McPherron, S. P., Alemseged, Z., Marean, C. W., Wynn, J. G., Reed, D., Geraads, D., Bobe, R., \& Bearat, H. (2010). Evidence for stone-toolassisted consumption of animal tissues before 3.39 million years ago at Dikika, Ethiopia. Nature, 466, 857-860. doi:10.1038 /nature09248

Morgan, T.J.H., Uomini, N.T., Rendell, L.E., Chouinard-Thuly, L., Street, S.E., Lewis, H. M., Cross, C.P., Evans, C., Kearney, R., de la Torre, I., Whiten, A., Laland, K.N. (2015). Experimental evidence for the co-evolution of hominin tool-making, teaching and language. Nature Communications, 6, 6029 doi.org/10.1038/ncomms7029

Nelson, K. (1998). Language in cognitive development. Cambridge: Cambridge University Press.

Searle, J. (1969). The construction of social reality. Cambridge: Cambridge University Press.

Tomasello, M. (2008). The origins of human communication. Boston, MA: MIT Press.

Toth, N., Schick, K., \& Semaw, S. (2003). A comparative study of the toolmaking skills of Pan, Australopithecus, and Homo sapiens. In N. Toth \& K. Schick (Eds.), The Oldowan: case studies of the earliest Stone Age. Bloomington, IN: Craft Press.

Whiten, A., Toth, N., \& Schick, K. (2009). The evolution and cultural transmission of percussive technology: integrating evidence from palaeoanthropology and primatology. Journal of Human Evolution, 57, 420-435.

Whiten, A. (2011). The scope of culture in chimpanzees, humans and ancestralapes. Philosophical Transactions of the Royal Society. B, Biological Sciences, 366, 997-1007, doi.org/10.1098 /rstb.2010.0334

Wrangham, R. (2009). How cooking made us human. New York: Basic Books. 